\title{
ESCENAS EN LA VIDA DE SRINAVASA RAMANUJAN: ENDEMIAS, EPIDEMIAS Y PANDEMIAS DESDE UNA MIRADA BIOMÉDICA Y CRÍTICA
}

\section{Scenes of Srinavasa Ramanujan's life: endemics, epidemics and pandemics from a biomedic and a critic point of view}

Ianina MORETTI BASSO ${ }^{1}$; Edgardo MORETTI REBOLA²; Beatriz BASSO IBAÑEZ ${ }^{2}$

1. Facultad de Filosofía y Humanidades, Universidad Nacional de Córdoba (Argentina).

2. Facultad de Ciencias Médicas, Universidad Nacional de Cordoba (Argentina).

Autora para correspondencia: Ianina MORETTI BASSO.

Correo electrónico: ianimoretti@hotmail.com ; i.kasiopea@gmail.com

Recibido: 27 de septiembre de 2020

Aceptado: 26 de octubre de 2020

\section{Resumen:}

El hombre que conocía el infinito / The man who knew infinity (2015) de Matt Brown, conjuga problemáticas de las que se ocupa este artículo. El filme se centra en la experiencia del matemático indio Srinavasa Ramanujan en Cambridge, donde sufre situaciones de racismo, discriminación, vivienda precaria, malnutrición. Aun así, logra que reconozcan su genio, siendo nombrado Miembro de la Royal Society of London. Durante su estadía, se desencadena la Primera Guerra Mundial y él enferma de tuberculosis. Sobre este contexto, el trabajo apunta a explorar afecciones que atañen a la humanidad toda: endemias, pandemias, guerra, discriminación. Se trata, de algún modo, de fenómenos, que afectan con intensidad a la salud y bienestar de poblaciones enteras. En ese marco, la visión crítica desde las humanidades permite preguntarse, si bien son eventos que afectan a toda una población, en qué sentido lo hacen diferencialmente respecto a ciertos sectores. Así, se considera el hecho de que hay cuerpos más expuestos, con mayor vulnerabilidad y menores condiciones para afrontar sus efectos. La producción de conocimiento científico no es ajena a esta distribución de la precariedad, como muestra el filme, y es preciso hacer foco en enfermedades desatendidas y de la precariedad en pos de generar condiciones sanitarias más igualitarias.

Palabras clave: pandemias; endemias; Srinavasa Ramanujan; precariedad; vulnerabilidad. 


\title{
ESCENAS EN LA VIDA DE SRINAVASA RAMANUJAN: ENDEMIAS, EPIDEMIAS Y PANDEMIAS \\ DESDE UNA MIRADA BIOMÉDICA Y CRÍTICA \\ IANINA MORETTI BASSO; EDGARDO MORETTI REBOLA; BEATRIZ BASSO IBAÑEZ
}

\begin{abstract}
The selected film, The man who knew infinity (2015), by Matt Brown, addresses issues which are fundamental to this article's objectives. The film is about Srinivasa Ramanujan's experience as an Indian mathematician at Trinity College (Cambridge) where he suffers racism, discrimination, poor housing conditions, malnutrition. Even so, he achieves recognition as a genius, and ultimately becomes an academic member of the College. During his stage in Cambridge, World War I begins, and he gets ill with tuberculosis. In this context, this article aims to explore affections that involve humanity as a whole, such as endemics, pandemics, war, discrimination, among others. It phocuses on phenomena that affect intensely entire populations' health and wellbeing. In this sense, a critical vision given by the humanities can question the senses in which these three notions affect a whole population, and yet affect it in different ways and intensities. Thus, this work underlines the fact that some bodies are more vulnerable, more exposed to precarization, and have less conditions to overcome its effects. Scientific production of knowledge is not an exception to this distribution of precariousness, as the film shows. In this light, it is necessary to refocus on neglected diseases and precariousness diseases, in order to create egalitarian conditions for prevention and medical treatment.
\end{abstract}

Key words: pandemics; endemia; Srinavasa Ramanujan; precarity; vulnerability.

\section{Ficha técnica}

Título: El hombre que conocía el infinito. Título original: The man who knew infinity. País: Reino Unido.

Año: 2015.

Director: Matt Brown.

Música: Coby Brown.

Fotografía: Larry Smith.

Montaje: JC Bond.

Guión: Matt Brown, Robert Kanigel (Novela: Robert Kanigel).

Intérpretes: Dev Patel; Jeremy Irons; Devika Bhise; Toby Jones; Stephen Fry; Jeremy Northam...

Color: color.

Produccción: Edward R. Pressman, con la colaboración de Matemáticos como Manjul Bhargava y Ken Ono.

Duración: 114 minutos.

Género: drama, biográfico.

Productoras: Animus Films, Edward R. Pressman Film, Firecracker Entertainment.

Sinopsis: El filme se centra en el período en que Srinivasa Ramanujan, proveniente de India, es admitido en el exclusivo Trinity College, de Cambridge, por quien sería su mentor, el matemático G. H. Hardy. Después de salvar situaciones con profesores y estudiantes ingleses muy conservadores e incluso una relación conflictiva con su protector, finalmente reconocen su genio y es propuesto como miembro académico del College; recién en un segundo intento aceptado como miembro de la Royal Society of London y reconocido como un pionero en teorías matemáticas. Durante su estadía en Cambridge se desencadena la primera guerra mundial y enferma de Tuberculosis. Ramanujan decide finalmente volver a su país, para reunirse con su esposa. En el viaje de regreso empeora su enfermedad, de la cual nunca puede recuperarse y fallece, a los 32 años. Hoy, sus fórmulas y teoremas se emplean para develar algunos misterios, como el de los agujeros negros (el infinito).

Enlaces: https://www.filmaffinity.com/ar/ film476371.html https://www.imdb.com/title/tt0787524/ 


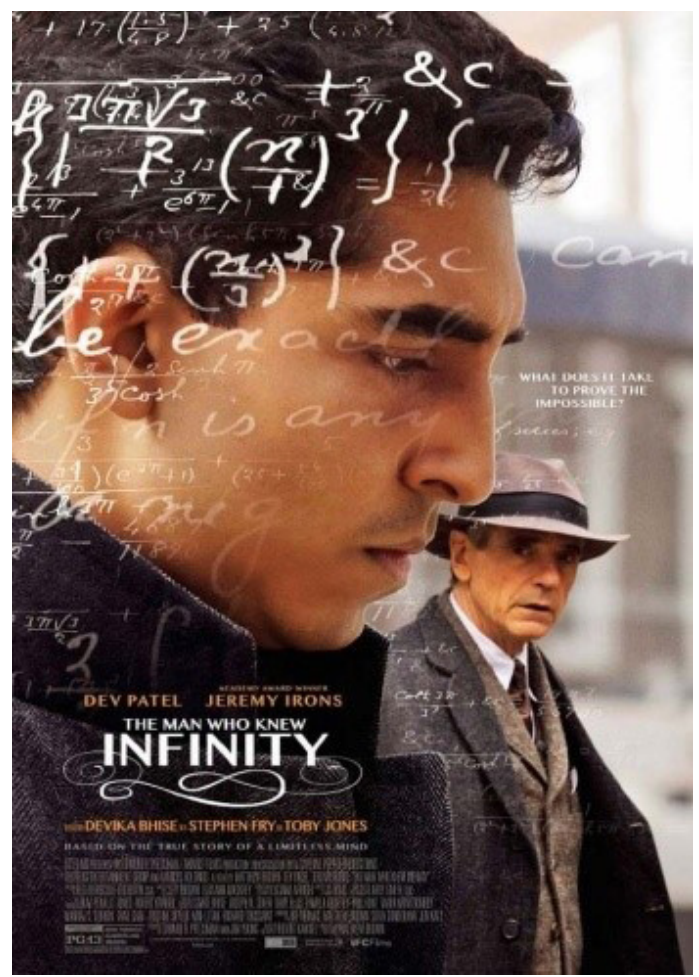

Cartel

\section{Introducción}

En el presente artículo se analiza el filme $E I$ hombre que conocía el infinito / The man who knew infinity (2015) de Matt Brown, en el cual se entrecruzan problemáticas sanitarias con modos de precarización material y estigmatización social. En el filme se representan escenarios trágicos para la humanidad toda: endemias, pandemias, guerra, discriminación. Se trata, de algún modo, de fenómenos que afectan con intensidad a la salud y bienestar de poblaciones enteras. Tal es el caso de las epidemias, noción que proviene del griego $\dot{\varepsilon} \pi \mathrm{l}-\delta \dot{\eta} \mu \mathrm{o}$ ऽ y significa "estar en su país, patria o casa» ${ }^{1}$, y las pandemias -de $\pi a v$ - $\delta$ ń $\mu$ to «que va por todo el pueblo o todos los países $»^{1}$-. Sin embargo, la perspectiva desde las humanidades permite preguntarse por el modo en que estos fenómenos afectan en principio de modo universal, y al mismo tiempo la forma particular en que repercute diferencialmente en las poblaciones ${ }^{2}$. Esto es, cómo es que, a pesar de afectar en principio «a todos», nunca el impacto es por igual.

La filósofa Judith Butler ha estudiado la vulnerabilidad de modo tal que nos permite distinguir entre, por un lado, la condición generalizada de vulnerabilidad común -en tanto todos somos cuerpos finitos y afectables-y la distribución diferencial y geopolítica de dicha vulnerabilidad ${ }^{a, 3}$. Esta distribución evidencia «aquello que políticamente induce una condición en la que cierta parte de las poblaciones sufren la carencia de redes de soporte social y económico, quedando marginalmente expuestas al daño, la violencia y la muerte ${ }^{4}$. Las poblaciones precarizadas se encuentran especialmente expuestas a las enfermedades, a la escasa prevención y deficiente atención médica. El filme nos muestra hasta qué punto las condiciones materiales, así como la etnia y el estatus social (en el caso del ámbito universitario), inciden en la vulnerabilidad de ciertos cuerpos, quedando así más expuestos a la enfermedad, la discriminación y hasta el abandono.

\section{Vida de Srinavasa Ramanujan anterior a lo tratado en la película}

La vida de Ramanujan, uno de los matemáticos más importantes en la historia de esta ciencia, permite analizar escenas en las que se intersecan condiciones de precariedad. Vivió expuesto a las afecciones de la época: enfermedades, pero

a «De algún modo, todos vivimos con esta particular vulnerabilidad, una vulnerabilidad ante el otro que es parte de la vida corporal, ante (...) lo que no podemos prevenir. Sin embargo, esta vulnerabilidad se exacerba bajo ciertas condiciones sociales y políticas», dice Butler, haciendo hincapié en poblaciones precarizadas, inmigrantes, discriminadas por su género, clase, etc. 


\section{ESCENAS EN LA VIDA DE SRINAVASA RAMANUJAN: ENDEMIAS, EPIDEMIAS Y PANDEMIAS \\ DESDE UNA MIRADA BIOMÉDICA Y CRÍTICA \\ IANINA MORETTI BASSO; EDGARDO MORETTI REBOLA; BEATRIZ BASSO IBAÑEZ}

también pobreza, dificultades por pertenecer a una colonia, y el racismo y la discriminación en un mundo signado por la guerra. Nació en 1887 en Erode, sur de la India, donde vivía muy pobremente. Apenas tenía dos años cuando una epidemia de viruela se desató sobre la región, y Ramanujan contrajo esta enfermedad, la primera de las que debió enfrentar en su corta vida. Probablemente, también contrajo la disentería causada por Entamoeba histolytica. En regiones emergentes, como India o países latinoamericanos entre otros, las parasitosis intestinales son frecuentes, asociadas a las malas condiciones sanitarias, las inmunodeficiencias causadas por la mala nutrición y su consecuente mayor susceptibilidad a infecciones ${ }^{5,6}$.

Desde pequeño, Ramanujan sintió fascinación por las matemáticas, lo que se fue transformando a lo largo de los años en una verdadera pasión. Fue una de las mentes más lúcidas del siglo XX. Ya de muy joven, obtuvo una beca para estudiar en el Goverment College de Kumbakonam, pero, concentrado en las matemáticas, no aprobó las demás materias y perdió la beca. En 1906, se trasladó a Madrás, cuando era centro administrativo de la colonia británica. Se inscribió en la Universidad de Pachaiyappa y, nuevamente, su obsesión por el estudio de las matemáticas le impidió ingresar y sus profesores descreían de él.

Como su familia, era devoto de una deidad, la diosa Shree Lakshmi Namagiri, por lo cual gran parte de su vida transcurría alrededor del templo, orando, o bien escribiendo fórmulas matemáticas a las que no le conocía su aplicación, por la falta de educación superior. Sus padres arreglaron el matrimonio con Janaki de tan solo 9 años de edad.

\section{Versión cinematográfica}

La película nos permite analizar en diferentes detalles los modos en que la producción científica de Ramanujan estuvo atravesada por su experiencia vital y la precariedad de las condiciones materiales y emocionales en las que trabajó en Inglaterra, como un llamado de atención sobre las afecciones muchas veces desatendidas de la salud en sentido amplio. El filme comienza cuando Ramanujan es un joven, ya casado, que debe vivir separado de su esposa Jamaki, él en un pabellón de solteros y ella en casa de la madre de Ramanujan. No tenían un hogar para habitar juntos. Por ello buscaba desesperadamente conseguir trabajo, lo cual también era requerido por su esposa. En esta búsqueda, conoce a un recaudador de impuestos de la administración colonial, quien descubre sus inéditas y extraordinarias condiciones para las matemáticas. Amante de esta disciplina, le ofrece trabajo en el Madrás Port Trust a cambio que le enseñe matemáticas por las noches, lo que es inmediatamente aceptado por Ramanujan y de esa forma, le permite conseguir una modesta vivienda para cohabitar con Janaki y su madre. Para vislumbrar mejor las condiciones de vida de Madrás, un hecho de no menor importancia es que este precoz genio usaba grandes cantidades de papel, donde desarrollar sus fórmulas y ecuaciones, sin embargo, hasta este insumo le era racionado y en la oficina británica le ordenaron que utilizara papel para embalaje.

El funcionario consigue convencerlo de que hay un mundo más allá de Madrás, que debía ir a Inglaterra para su perfeccionamiento y hacerse conocer en el ambiente científico. Para ello, consigue el visto bueno del Departamento de Matemáticas del Trinity College, Cambridge, específicamente de un matemático que se ofrece a ser su tutor, Godfrey Harold Hardy. Antes de aceptarlo, Hardy intercambia opiniones con su discípulo, John E. Littlewood, sobre las posibles dificultades que le ocasionaría traer a la Universidad de Cambridge a alguien proveniente de la colonia, por la segura discriminación racial a que sería sometido y las dificultades que le ocasionaría al propio Hardy. Finalmente, su amor a la ciencia matemática lo indujo a enfrentar esos prejuicios a cambio de 


\section{ESCENAS EN LA VIDA DE SRINAVASA RAMANUJAN: ENDEMIAS, EPIDEMIAS Y PANDEMIAS \\ DESDE UNA MIRADA BIOMÉDICA Y CRÍTICA \\ IANINA MORETTI BASSO; EDGARDO MORETTI REBOLA; BEATRIZ BASSO IBAÑEZ}

conocer a quien podría ser un genio, según las ecuaciones y fórmulas que le fueron enviadas en el pedido de aceptación.

La madre del joven se opone al viaje, pues en Madrás la religión prohíbe atravesar el océano, pero Janaki lo apoya y alienta, pidiéndole que luego la lleve a ella. Antes de partir, la madre le pide que no se contamine con la comida de los ingleses, también por motivos religiosos y, como se verá más adelante, Ramanujan obedecerá hasta sus últimas consecuencias.

Cuando llega a Cambridge, se queda absolutamente atónito e inmovilizado por la magnificencia de los edificios de la Universidad; es probable que en ese momento una corriente eléctrica haya recorrido su cuerpo de la cabeza a los pies, y su mente haya volado a su querido, lejano y pobre Madrás y se le haya cruzado una pregunta ¿Qué hago yo aquí? Y, sin embargo, allí está, desafiando todos los impedimentos que su origen indio suponían para los estándares sociales de aquella época. Allí lo espera Littlewood, quien lo acompaña alrededor de los jardines, muy bien cuidados y cuando Ramanujan en un momento pisa el césped, una voz severa le manifiesta airadamente que no lo haga nunca, porque ese espacio sólo lo pueden pisar los académicos ${ }^{\mathrm{b}}$.

Bajo ese estado anímico y emocional, por ese mundo nuevo que se abre a sus ojos aldeanos, llega a la oficina de Hardy. El choque de culturas con su tutor aflora desde el primer momento, pero ambos se necesitan mutuamente, Ramanujan para publicar sus fórmulas absolutamente inéditas y el profesor debido a su pasión por las matemáticas, intuyendo que está frente a un verdadero genio, un diamante en bruto (Foto 1 ).

b El mismo césped que muchos años atrás también le habían prohibido atreverse a pisarlo a la escritora Virgina Wolf (V. Wolf. Un Cuarto propio. 1a Ed. Booket, Grupo Edit. Planeta Mexicana. Ciudad de México 2019, pág. 14-15).

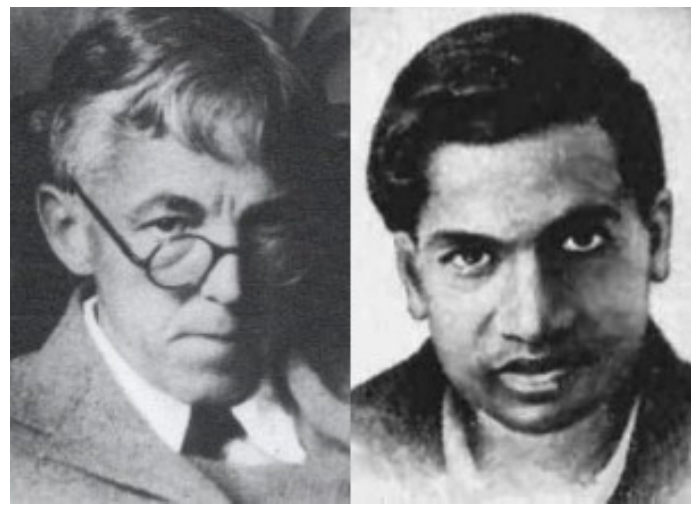

La cambiante relación entre Hardy y Ramanujan es el hilo conductor de la película. Las matemáticas son la excusa para enlazar, no sólo la relación de los protagonistas, sino otros temas que confluyen, como el racismo en el mundo académico, las diferencias culturales, el ego y el miedo a lo desconocido 7 . Hardy es extremadamente conservador y estructurado, por lo cual no concibe que los conocimientos de Ramanujan no provengan de una educación formal y le exige tomar clases de Matemáticas. Ocurre entonces una escena dramática, en la cual un profesor expone un teorema muy delicado y Ramanujan lo resuelve inmediatamente. El profesor no soporta lo que considera una humillación, por lo cual lo fustiga con una muy fuerte reprimenda, echándolo y prohibiendo su regreso a tomar más clases. De esta manera se pone de manifiesto la discriminación racial, pues el profesor no acepta que un extranjero e indio lo supere intelectualmente, y al mismo tiempo se deja ver la estricta jerarquía que funcionaba en la academia. En ese marco, la inteligencia de Ramanujan parecía ser más una amenaza para la jerarquía establecida que una buena noticia, sólo concebida por Hardy aún a pesar de su propio racionalismo estricto.

Como un alivio a su situación anímica, se refugia en su religión, incluso armando un pequeño altar en su dormitorio, donde reza diariamente, lo cual le da fuerzas para seguir enfrentando las 


\section{ESCENAS EN LA VIDA DE SRINAVASA RAMANUJAN: ENDEMIAS, EPIDEMIAS Y PANDEMIAS \\ DESDE UNA MIRADA BIOMÉDICA Y CRIITICA \\ IANINA MORETTI BASSO; EDGARDO MORETTI REBOLA; BEATRIZ BASSO IBAÑEZ}

adversidades por las que atraviesa y, en lo específicamente referido a su trabajo, las constantes disputas con Hardy. El profesor insiste en pedirle «pruebas» sobre las conclusiones de Ramanujan quien, en cambio, se ve urgido por publicar. En un intento por convencerlo acerca de la importancia de «dejar un legado», Hardy conduce a Ramanujan a la Biblioteca del Trinity College. Según el Profesor, falta mucho camino por recorrer, agregando método y disciplina al conocimiento intuitivo del joven. Para motivarlo, le muestra en una vitrina un libro de Newton, quien fue uno de los alumnos más destacados del College, comparándolo incluso con el sabio y augurando que algún día allí se expondrían sus trabajos.

En lo que respecta a su vida en Cambridge, debido a la prohibición materna de contaminarse con las comidas británicas, no concurre al comedor universitario, alimentándose pobremente en su dormitorio. El frío, el clima húmedo de Inglaterra, la mala alimentación y la falta de afectos familiares provocan en este joven genio una creciente debilidad física y emocional. Extraña noticias de su esposa, quien, aparentemente, no responde sus cartas $^{8}$. La madre de Ramanujan, sin medir el daño físico, espiritual y moral de la pareja, intercepta las cartas que su hijo escribe a su esposa y no envía la correspondencia de Janaki. Ramanujan, mente privilegiada, se apoya en su religión y en sus convicciones, sacrificando todo lo demás en función de su objetivo.

Todo ese sufrimiento no es observado por Hardy, quien ve a Ramanujan como a un productor de geniales descubrimientos, sin considerar quizá lo más importante para su protegido en esos momentos, su faz espiritual y humana. Coincidente con estas observaciones en el artículo de Cine y psicología, se expresa que el profesor ingles solo le «ve» "su potencial matemático», lo «ve» como una «inversión» para las matemáticas, pero no "ve" el ser humano que es portador de ese potencial ${ }^{9}$. Las afecciones de Ramanujan pasan inadvertidas.
En un momento, entra en escena el filósofo Bertrand Russell ${ }^{c}$, catedrático en el Trinity College quien, probablemente por su formación humanista en varias ocasiones critica severamente a Hardy por el estricto trato que le da a Ramanujan y lo insta a que lo deje más libre, pues «cada uno tiene sus propios tiempos». Esta conversación moviliza los sentimientos del estructurado matemático y, al quedar solo, comienza a reflexionar sobre su comportamiento frente a su pasante. También influye LIttlewood, un hombre más sensible, quien le advierte a Hardy antes de partir hacia la guerra: "tienes que cuidar de él. Tú también tienes una guerra que librar, pero no dejes que sea contra Ramanujan»?.

Simultáneamente a estos acontecimientos académicos, científicos y humanos en la vida del joven, se desencadena la Primera Guerra Mundial. Durante los preparativos para la guerra, se manifiesta en forma cruda, nuevamente, la discriminación, cuando compañeros universitarios voluntarios golpean duramente a Ramanujan por su condición de extranjero ("este Indio se queda cómodamente acá mientras nosotros marchamos a morir en la guerra»). Otra vez vemos cómo se intersecan las condiciones de vulnerabilidad de Ramanujan, exponiéndolo de modo diferencial a la violencia, incluso de sus propios compañeros.

En una fuerte discusión Hardy interpela a Ramanujan sobre el origen de sus conocimientos, a lo que él responde: "las ideas vienen de mi diosa Shree Lakshmi Namagiri, que me habla cuando duermo. Una ecuación no tiene sentido si no expresa un pensamiento de Dios». Se ponen de manifiesto en este momento muy claramente las diferencias culturales y religiosas. El secularismo y racionalismo se ven enfrentados a la religiosidad y la intuición. La idea de progreso, como marca de la modernidad occidental y de un modo de comprender la ciencia, intenta erigirse como la

c Más tarde Russell sería expulsado del Trinity College por oponerse expresamente a la Guerra. 


\section{ESCENAS EN LA VIDA DE SRINAVASA RAMANUJAN: ENDEMIAS, EPIDEMIAS Y PANDEMIAS \\ DESDE UNA MIRADA BIOMÉDICA Y CRÍTICA \\ IANINA MORETTI BASSO; EDGARDO MORETTI REBOLA; BEATRIZ BASSO IBAÑEZ}

contrapartida de la religión. Sin embargo, ¿qué principios entraban allí en juego? ¿cuáles eran las verdaderas razones de rechazar lo religioso? Por momentos el filme deja entrever otra cantidad de dogmas y jerarquías funcionando, en nombre del secularismo, que se reclama como único modo posible de lo contemporáneo². La religión por ende aparece juzgada como algo del pasado, que «atrasa» o es signo del no-saber. No obstante, una escena abre otra posibilidad, manifiesta otro modo de relación entre ciencia y religión. Hardy manifiesta «no puedo creer en Dios, pero creo en ti». Es un momento clave del filme: dos cosmovisiones que se permiten conversar y enlazarse en la producción de conocimiento.

Hardy intenta que Ramanujan tenga éxito, por razones diversas: su amor a la ciencia, su propio ego y la demostración ante sus colegas de que no se equivocó al traer a un extranjero a Cambridge. En un momento logra que se publique el primer trabajo de Ramanujan, quien al fin muestra una expresión parecida a la felicidad en su rostro sombrío. Su tutor, al comprobar la genialidad del joven, hace su primer intento de que sea incorporado como Miembro Académico del Trinity College, pero desgraciadamente existe un racismo tan marcado también en el ámbito científico, donde se suman los egoísmos y celos, que esta solicitud es categóricamente denegada. La sociedad lo expone a la violencia, padece agresiones en el ámbito universitario, de compañeros y profesores, sin embargo, él los enfrenta con sus ecuaciones y sus fórmulas matemáticas, sin responder a las agresiones, al contrario, tratando de sustraerse de ellas.

A pesar de las diferencias que los separan, ambos tutor y pasante siguen trabajando juntos, "full time», en Particiones, Números Primos, Serie infinitas, nada los detiene. Otro momento crucial es el desafío al que lo somete Hardy con una autoridad del College sobre las particiones. Ramanujan resuelve en pocos minutos y un par de páginas lo que al profesor le ha llevado días e infinidad de cálculos. Es un triunfo fundamental, que comienza a cambiar la percepción que tiene el cuerpo docente de Cambridge sobre el joven genio. Así, Hardy logra en un segundo intento y luego de una disertación emotiva y científicamente incontrastable, que lo designen miembro de la Royal Society of London.

Finalmente, por las condiciones ya descritas respecto a clima, enfermedades de época y condiciones físicas y emocionales, Ramanujan enferma gravemente y debe ser internado. Se le diagnostica tuberculosis. Posiblemente, como se verá más adelante, complicada con una secuela de la amebiasis. Con elevada fiebre, delira y huye del hospital con la decisión de quitarse la vida, arrojándose sobre los rieles del tren. Milagrosamente, el maquinista logra frenar y salvarlo. Nuevamente es internado en el hospital, donde un día, Hardy le lleva una carta de la India. La misiva es de su esposa y, al leerla con desesperación, se entera que ella se ha ido a vivir con su hermano, por la pésima relación con su suegra y la falta de noticias de él, que, durante años, aparentemente, no había respondido sus cartas. Ramanujan queda anonadado, y con su fiebre y accesos de tos no puede hablar. Hardy lo acompaña y desde ese momento se dirige a él con actitud paternal, pidiéndole que se cure pronto, que deben seguir trabajando. Su rostro solo se ilumina cuando se habla de números, ya que Ramanujan siente amor por ellos, a los que le encuentra «una belleza intrínseca». En otra visita, Hardy lo encuentra muy desmejorado y, no sabiendo qué decir, le cuenta que el número del taxi donde había viajado tenía una matrícula poco atractiva, 1729, a lo cual Ramanujan responde: "No, es muy interesante, pues es el número más pequeño que se puede expresar en la suma de dos cubos positivos en dos formas diferentes: 1729= $1^{3}+12^{3}=9^{3}+10^{3} »$. Desde entonces, este número es denominado Taxicab y conocido como «número de Hardy-Ramanujan $»^{10,11}$. Otra subliminal discriminación, pues debería ser llamado en orden inverso, en honor a su real descubridor. 


\section{ESCENAS EN LA VIDA DE SRINAVASA RAMANUJAN: ENDEMIAS, EPIDEMIAS Y PANDEMIAS \\ DESDE UNA MIRADA BIOMÉDICA Y CRÍTICA \\ IANINA MORETTI BASSO; EDGARDO MORETTI REBOLA; BEATRIZ BASSO IBAÑEZ}

En este contexto, Ramanujan toma la resolución irrevocable de volver a Madrás: «La guerra ha acabado, vuelvo a casa». En viaje de regreso a su país tiene una recaída muy severa en su salud, de la cual no logra recuperarse. Aún puede vivir poco más de un año con su esposa y con una producción científica acorde a su mente prodigiosa, a pesar de encontrarse al borde de la muerte, que finalmente ocurre a sus 32 años. La obra de Ramanujan va a trascender el tiempo y va a abrir caminos para posteriores investigaciones en la matemática. Sus ecuaciones se usan hoy en día, entre muchas otras aplicaciones, para descifrar los misterios de los agujeros negros del cosmos ${ }^{12}$.

Enfermedades endémicas, epidémicas y pandémicas en la época de Ramanujan y hasta el presente

Las siguientes enfermedades fueron parte del paisaje sanitario en la época de Ramanujan, a algunas de las cuales se vio expuesto, en mayor proporción por las condiciones de vida y contexto. Por otra parte, afecciones de aquel momento histórico aún aquejan a la humanidad, y en especial a poblaciones precarizadas.

\section{Viruela}

Es una de las plagas que azota a la humanidad desde tiempos antiguos. Un salto cualitativo fundamental en la lucha contra esta enfermedad la dio Edward Jenner, a partir de 1796, cuando descubre los rudimentos de una vacuna desde una aguda observación y sin conocer todavía la existencia de los microorganismos. Recién en 1840 el gobierno británico autorizó la vacunación gratuita contra la viruela. Sin embargo, la misma distaba de ser masiva.

\section{Parasitosis intestinales}

Un documental sobre la vida de Ramanu$j^{12}{ }^{12}$ y una escena esbozada en el presente filme, sugieren que de la amebiasis nunca se curó y que podría haber provocado un absceso hepático, la complicación extraintestinal más importante de la amebiasis. El absceso hepático amebiano es una entidad cuya prevalencia es mayor en zonas tropicales y en áreas con deficientes condiciones sanitarias, pero se manifiesta recién en la adultez. Los síntomas más destacados son dolor en cuadrante superior derecho (que se observa en la película, cuando Ramanujan tose y se toca desesperadamente el flanco derecho), fiebre e importante afectación del estado general ${ }^{13}$.

\section{Tuberculosis}

Producida por Mycobacterium tuberculosis, es una enfermedad transmisible que constituye una importante causa de morbilidad, una de las 10 primeras causas de mortalidad del mundo y la principal debida a un solo agente infeccioso. Se estima que aproximadamente 10 millones de personas enfermaron de tuberculosis en 2018, un número que se ha mantenido relativamente estable en los últimos años ${ }^{14}$.

En el cine, la tuberculosis ha sido quizás la enfermedad más atractiva, como lo demuestran las más de 400 películas citadas por Garcia Sanchez y col: «Hoy el séptimo arte sigue llamando la atención sobre la peste blanca, en no pocas películas recientes la tuberculosis se desliza por sus argumentos» ${ }^{15}$. Incluso ha tenido un halo de romanticismo (La Dama de las Camelias, La Traviata, entre otras). El Hombre que conocía el infinito cuenta que, lamentablemente, la tuberculosis tronchó precozmente la vida de un genio como S. Ramanujan. La literatura también fue vertiente de esta enfermedad, tanto en las obras como en los escritores que la padecieron ${ }^{16}$.

En aquella era preantibiótica, el único paliativo lo constituía quizás el aire puro, que por ejemplo en Argentina se encontraba en las serranías cordobesas, en las cuales se construyeron hospitales exclusivamente para internación de pacientes tuberculosos y que también dio lugar a un filme basado en la novela de Manuel Puig, Boquitas pintadas $^{15}$. Desde luego esas condiciones no 


\section{ESCENAS EN LA VIDA DE SRINAVASA RAMANUJAN: ENDEMIAS, EPIDEMIAS Y PANDEMIAS \\ DESDE UNA MIRADA BIOMÉDICA Y CRÍTICA \\ IANINA MORETTI BASSO; EDGARDO MORETTI REBOLA; BEATRIZ BASSO IBAÑEZ}

estaban dadas en el húmedo y frío clima inglés ni tampoco en Madrás. Ello sumado, como se mencionó anteriormente, a la mala nutrición y al estrés fueron fatales para Ramanujan. De acuerdo al documental citado ${ }^{12}$ podría especularse que habría presentado una comorbilidad en el sentido de un absceso hepático por amebiasis. En estos momentos, si bien se encuentran antibióticos disponibles para esta patología, no todos los pacientes tratados curan, debido a la emergencia de cepas resistentes de $M$. tuberculosis.

Otras endemias y pandemias que coexistieron en la vida de Ramanujan, aunque no reflejadas en el filme fueron la malaria y la mal llamada gripe española, entre 1918 y 1920. Esta última considerada una de las pandemias más devastadoras que sufrió la humanidad.

Desde ese dramático ayer a una actualidad dolorosa

Desde tiempos remotos, existieron plagas que afectaron a la humanidad, con mayor incidencia en poblaciones vulnerables. Estas enfermedades son más prevalentes en países tropicales y con escasos recursos económicos. Entre ellas, algunas fueron específicamente clasificadas en la categoría de «enfermedades desatendidas» por OMS, como por ejemplo Chagas en Latinoamérica ${ }^{17}$, dengue, esquistosomiasis, entre otras. Asimismo, otras enfermedades, como la tuberculosis, afectan diferencialmente según la posibilidad de acceso a los servicios de salud.

Hoy, el mundo se encuentra atravesando una grave pandemia, por un Coronavirus, cuyo origen fue en China, a finales de 2019. Hasta el presente, se han producido más de 30 millones de infectados y alrededor de un millón de muertes ${ }^{18}$. Si con los actuales conocimientos y el avance científico tecnológico, la pandemia de Covid-19 aun dista de ser controlada, a pesar de que diferentes vacunas ya se encuentran en fase 3 , podremos imaginar lo que significaban las plagas en épocas en las cuales toda la batería de medicamentos y procedimientos actuales eran desconocidos.
El análisis final del presente filme permite abordar algunos aspectos fundamentales y remarcar lo importante que fue, y en algunos casos sigue siendo, la prevención, el diagnóstico precoz y el tratamiento de las enfermedades anteriormente mencionadas, las cuales podrían tener una mejor evolución de contar con estas acciones sanitarias.

En este marco, hoy se vuelve a vislumbrar la vulnerabilidad humana, universalmente compartida, pero a la vez, diferencialmente distribuida. Las poblaciones más precarizadas son las menos preparadas para enfrentar las complejidades de afecciones como las que muestra el film y aquí se recuperan. En el caso de la pandemia, nos habilita a pensar hoy que, si bien el virus no distingue clases ni razas ni género, la diferencia está signada por la posibilidad o no de contar con condiciones para prevenir el contagio y, de contraer la enfermedad, por las condiciones de atención médica. La vivienda, por ejemplo, es hoy un derecho incontestable para poder cumplir con la consigna del resguardo en cuarentena; del mismo modo, la posibilidad de acceder al sistema de salud es fundamental. Sin embargo, dichas condiciones no están garantizadas de igual forma para todos. Es así que, al analizar las enfermedades, al abordar críticamente sus efectos, es menester atender a esta distribución diferencial de la precariedad, marcando a unas poblaciones como más vulnerables que otras ${ }^{19}$. Por eso también es relevante preguntarse cuáles enfermedades siguen siendo desatendidas, o menos atendidas, las que afectan a qué poblaciones. El racismo y la relación poscolonial siguen marcando la distribución geopolítica de la precariedad, como lo mostraba ya el filme sobre Ramanujan. Sin embargo, el filme muestra también cómo el saber se abre paso en una comunión, un hacer colectivo entre el conocimiento de Ramanujan y la metodología y la institución que habilita Hardy; una comunión entre cosmovisiones, una alianza entre culturas, que se erige, precaria, ante las plagas que azotaban su mundo. 


\section{ESCENAS EN LA VIDA DE SRINAVASA RAMANUJAN: ENDEMIAS, EPIDEMIAS Y PANDEMIAS \\ DESDE UNA MIRADA BIOMÉDICA Y CRÍTICA \\ IANINA MORETTI BASSO; EDGARDO MORETTI REBOLA; BEATRIZ BASSO IBAÑEZ}

\section{Referencias}

1. Pabón $\mathrm{S}$ de Urbina J. M. Diccionario Manual Griego - Español VOX, 17ma Ed. Barcelona: Bibliograf; 1991.

2. Moretti Basso, I. Cuerpos y alianzas. Un estudio butleriano sobre las posibilidades performativas de la agencia frente a la violencia normativa. Tesis Doctoral en Filosofía. Córdoba: Facultad de Filosofía y Humanidades, Universidad Nacional de Córdoba; 2020.

3. Butler, J. Vida precaria. El poder del duelo y la violencia. Buenos Aires: Paidós; 2006.

4. Butler, J. Performatividad, precariedad y políticas sexuales. AIBR [Internet]. 2009. 4(3): 321-36.

5. Petri WA Jr, Mondal D, Peterson KM, Duggal P, Haque R. Association of malnutrition with amebiasis. Nutr Rev. 2009;67( Suppl 2):S207-S215.

6. Ibrahim MK, Zambruni M, Melby CL, Melby PC. Impact of Childhood Malnutrition on Host Defense and Infection. Clin Microbiol Rev. 2017;30(4):91971.

7. Federación de Entidades Profesionales Universitarias de Tucumán. Colegio De graduados En Ciencias Económicas de Tucumán. Cine Debate - El hombre que conocía el infinito (2015) 8vo ciclo [Internet]. 2016.

8. Tomás y Garrido G. El hombre que conocía el infinito. Cine desde el espectador. Asociación Española de Bioética y Etica Medica [Internet]. Granada 29 de noviembre de 2016.
9. Cardona J. El hombre que conocía el infinito (Matt Brown, 2015): De la razón y la intuición Cine y psicología [Internet]. 4 de agosto de 2018.

10. Jimenez A. El número de Hardy-Ramanujan. Xataka Ciencia [Internet]. 12 Septiembre 2006.

11. López Pellicer M. Ramanujan: Matemático genial desde la pobreza extrema. Rev R Acad Cienc Exact Fis Nat. (Esp). 2014;107(1-2):43-54.

12. Ramanujan, el matemático y su legado. Documental Open Frame Film Festival. 2016.

13. Fleta Zaragozano J, Bueno Lozano O, Bueno Lozano M. Amebiasis intestinal y absceso hepático amebiano. Med Integral (Ed. Impr). 2000; 36(3):96-101.

14. Organización Mundial de la Salud. Informe mundial sobre tuberculosis 2019.

15. García Sánchez J E, García Sánchez E, García Merino $E$. Tuberculosis y cine. Una aproximación a través de la fantasía de más de 400 películas. Rev Med Cine 2010; 6(3): 91-346.

16. Fresnadillo Martínez M J. Las enfermedades infecciosas en la literatura. Una larga historia sin final. Rev Med Cine 2015; 11(1): 41-53.

17. Basso B, Moretti E. Chagas. De exótica patología tropical a enfermedad globalizada. Rev. Med Cine, 2012; 8(1):4-11.

18. WHO. Coronavirus disease (COVID-19) pandemic.

19. Moretti I, Perrote N. Sentirse precarias. Afectos, emociones y gobierno de los cuerpos. Córdoba: Editorial Universidad Nacional de Córdoba; 2019.

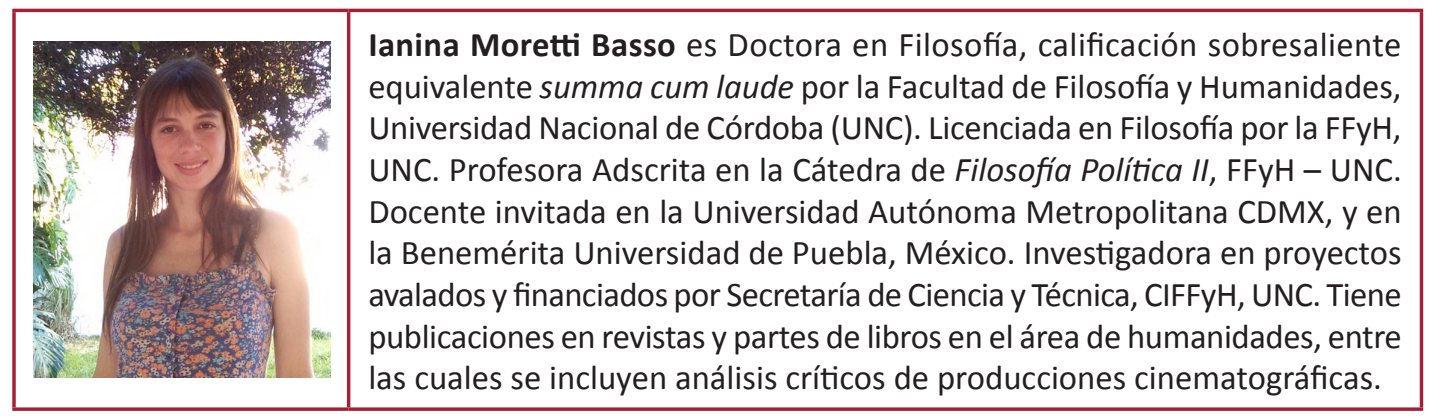

Rev. Med. Cine. 2021; 17 (2), 133-143 Ediciones Universidad de Salamanca / ஒ® J. Med. Mov., 2021; 17 (2), 133-143 [ 142 ] 


\begin{tabular}{|l|l|}
\hline & $\begin{array}{l}\text { Edgardo Moretti Rébola es Doctor en Bioquímica y Especialista en Inmuno- } \\
\text { logía. Fac. Ciencias Quimicas, Universidad Nacional de Córdoba, Argentina. } \\
\text { Profesor Investigador Fac. Cs Médicas, Universidad Nacional de Córdoba y } \\
\text { Dep. Microbiología, Universidad Nacional de Río Cuarto. Jefe de Laboratorio, } \\
\text { Dirección Nacional de Enfermedades transmisibles por vectores. Investiga- } \\
\text { dor Visitante Michigan State University; USA. Universite Libre de Bruxelles, } \\
\text { Belgique; IMBECU-CONICET, Mendoza, Argentina. Consultor ad honorem en } \\
\text { Enf. De Chagas, Org. Panamericana de la Salud. Miembro Comité de Bioética } \\
\text { Clínica y Ética de la investigación, Hosp Univ Maternidad y Neonatología, } \\
\text { Universidad Nacional de Córdoba y Hospital Italiano de Córdoba. }\end{array}$ \\
\hline $\begin{array}{l}\text { Beatriz Basso Ibañez es Doctora en Bioquímica y Especialista en Bacteriolo- } \\
\text { gía, Universidad Nacional de Córdoba, Argentina. Profesora e Investigadora } \\
\text { en Fac Cs Medicas y Jefa del Laboratorio de Microbiología Hosp Univ Mater- } \\
\text { nidad y Neonatología (HUMN), Univ Nac Córdoba. Jefa del Laboratorio de } \\
\text { Parasitología Serv Nac de Chagas, Córdoba. Organizadora y Directora de la } \\
\text { Carrera de Especializacion en Bacteriología Clínica, Universidad Nacional de } \\
\text { San Luis. Investigadora visitante en Michigan State University, USA, Univer- } \\
\text { site Libre de Bruxelles, Belgique e IMBECU, CONICET, Mendoza, Argentina. } \\
\text { Integrante Comité Bioética Clínica y Ética en Investigación HUMN. Más de } \\
100 \text { artículos en Revistas Nacionales e internacionales. }\end{array}$ \\
\hline
\end{tabular}

